\title{
Imaging and spectroscopy through plasmonic nano-probe
}

\author{
Y. Saito ${ }^{1, a}$ and P. Verma ${ }^{2,3}$ \\ 1 Frontier Research Center, Osaka University, 2-1 Yamadaoka, Suita, 565-0871 Osaka, Japan \\ 2 Department of Frontier Bioscience, Osaka University, 2-1 Yamadaoka, Suita, 565-0871 Osaka, Japan \\ 3 Department of Applied Physics, Osaka University, 2-1 Yamadaoka, Suita, 565-0871 Osaka, Japan
}

Received: 24 March 2009 / Accepted: 25 March 2009

Published online: 15 April 2009 - (c) EDP Sciences

\begin{abstract}
This article is a comprehensive review of the subject of near-field nano imaging and spectroscopy by surface plasmon polaritons induced on a metallized probe. The emphasis of the review is on fundamental aspects of plasmon enhancement and near-field spectroscopy, which are categorized as optical antenna structures, polarization properties in near-field, resonance frequency of surface plasmons, etc. Most recent studies that contribute to the understanding and interpretation of these phenomena are highlighted. Applications of near-field optics as newly established analytical tools for biology, materials sciences and plasmonic superlenses are included in the article.
\end{abstract}

PACS. 07.79.Fc Near-field scanning optical microscopes

\section{Introduction}

The wavelength range of the light visible to human eyes is about $400 \sim 700 \mathrm{~nm}$. The classical optical microscopes work best in this wavelength range, however, the resolving powers of these microscopes are limited by the wave nature of light [1]. Until recently, optical microscopy provided an image with resolution $\sim 500 \mathrm{~nm}$ in the visible wavelength of light. While there have been attempts to overcome this diffraction limit by using nonlinear response of materials $[2,3]$, near-field optics has immerged as an indispensable tool for sub-wavelength imaging that could provide better detecting accuracy [4-6]. In 1928, Synge proposed the idea of near-field scanning optical microscope (NSOM) [7], which can overcome the resolution of a conventional optical microscope where the resolution is limited by "diffraction limit" formulated by Abbe in 1873 [1]. Since the first proposal of Synge until the invention of scanning tunneling microscope (STM) [8], NSOM was not utilized experimentally, especially in the visible region of light, because of the difficulty in controlling the probe close enough to a sample surface, typically around $10 \mathrm{~nm}$. With the aid of the STM invention, scanning probe microscope technique matured, and in 1984, Pohl et al. reported the fundamental technique of NSOM [4], where they used an aperture type probe coated with thin metal films. Following this, NSOM has been exploited for many applications, such as spectroscopy and optical memory, by using an aperture type probe $[9,10]$. The early stage of NSOM has accessed the chemical properties of samples mainly by

\footnotetext{
a e-mail: yuika@ap.eng.osaka-u.ac.jp
}

fluorescence spectroscopy. Fluorescence imaging of a single molecules, using NSOM was first reported by Betzig [11], and the dynamics, such as fluorescence lifetime of a single molecule was studied by Xie and Dunn [12], and Ambrose et al. [13].

The resolution of an aperture type probe can go beyond the diffraction limit, however, it was still limited by the aperture size $(\sim 100 \mathrm{~nm})$. In recent years, apertureless type probes, such as a cantilever of atomic force microscope (AFM) [14], a metallic probe of STM [15], and a small metal particle [16], have been exploited for the achievement of much higher spatial resolution. The potential for improved spatial resolution is tremendous because an apertureless probe may be approximated as one or a few dipoles that interact locally with a sample surface [17]. The development of apertureless NSOM (ANSOM) using a metallic probe presents another advantages besides spatial resolution. The high throughput of the technique makes it feasible to perform the low sensitivity measurement. Two-photon fluorescence microscopy was presented in 1999 [18] and vibrational spectroscopy, such as Raman spectroscopy, which provides detailed information on molecular properties through vibrational energy detections, was also combined with NSOM technique in the year $2000[19,20]$. The cross-sections of Raman scattering $\left(\sim 10^{-30} \mathrm{~cm}^{-2}\right)$ is much smaller than that of IR absorption $\left(\sim 10^{-19} \mathrm{~cm}^{-2}\right)$ or fluorescence $\left(\sim 10^{-16} \mathrm{~cm}^{-2}\right)$. Therefore, signal-enhancement technique is very useful for Raman scattering measurements. Raman scattering from molecules adsorbed on metallic nanostructures is strongly enhanced due to the excitation of localized surface plasmon polaritons. This phenomenon is known as 


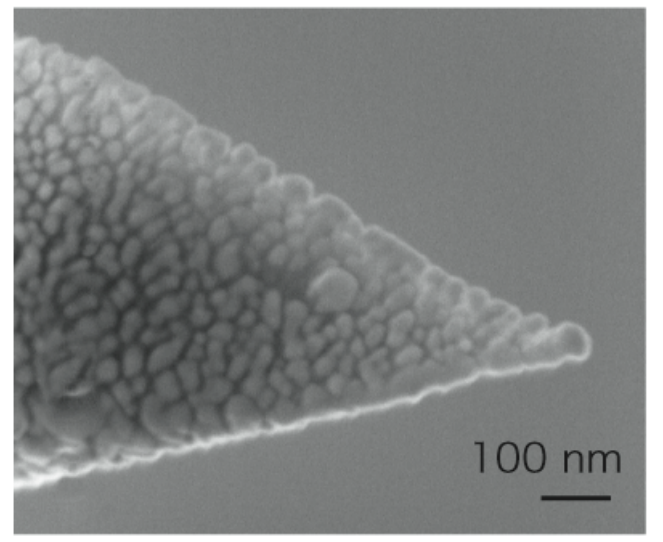

Fig. 1. Scanning electron microscope image of a silver coated near-field probe.

surface enhanced Raman scattering (SERS), the enhancement factor of which has been reported to reach up to $10^{5} \sim 10^{20}[21]$. The inclusion of a metallic probe tip in a system based on SERS, yields encouraging results as the probe can act as a nano-light source.

In this review, we begin by introducing the basic experimental configurations of ANSOM in Section 2 to clarify the concept of the technique. This will follow by sections classifieds in terms of enhancement mechanisms and physical effects. Section 3 is dedicated to plasmonic antenna structures and Section 4 is described the polarization properties in near-field experiments, and in Section 5, the resonance frequency of surface plasmons is described. In Section 6, we will briefly summarize the recent application of NSOM techniques to biology and single molecule detections. Plasmonic superlens effect by negative reflections will also be introduced, since the method has significant correlation to the NSOM imaging in the past few years. In this review, we try to include the most recent works mainly after the year 2004. Detailed reviews on earlier works can also be found in references $[22,23]$.

\section{Experimental configuration of ANSOM}

The experimental setup for ANSOM usually contains a metallic nano-probe, often in the form of a tip, which assists optical imaging through the enhancement and confinement of the light field due to the excitation of localized surface plasmons. Figure 1 shows the scanning electron microscopy (SEM) image of a silver coated probe prepared by vacuum evaporation method. The tip apex can be approximated as a small nano particle with size $\sim 30 \mathrm{~nm}$.

The experimental setup for ANSOM can be classified into two families, namely, "transmission-mode" (Fig. 2a) and "reflection-mode" (Fig. 2b). Transmission-mode has been widely used in ANSOM because in this mode the metallic tip is illuminated through a sample plane by an inverted microscope objective lens, which is highly efficient in both illumination and collection, especially when using a high numerical aperture $(N A)$ objective lens [19,20,24-26]. The advantage is particularly enormous for Raman spectroscopy, where the scattering cross section is small. Above all, evanescent illumination is possible for reducing the background signal by rejecting the small- $N A$ component $(N A<1)$ of the incident light [19]. However, transmission-mode ANSOM is not applicable to opaque or thick samples, as the incident light has to pass through the sample. In a reflection-mode, the tip is illuminated directly from the same side of the tip [27-31], which is advantageous and promising for observing opaque samples. In addition, an important requirement for attaining efficient enhancement under the tip is the polarization of the electric field of the incident light along the tip axis (see Sect. 4 for details). This condition is difficult to achieve in the transmission-mode but one can easily achieve it in the reflection-mode geometry. The problem with the reflection-mode is the interference of an AFM scanner, which prevents the access of the objective lens. Measurement with small $N A$ objective is necessary, which suffers from the low efficiency in both illuminations and collections.

\subsection{Transmission mode}

The configuration of transmission mode setup was first introduced by two groups independently in the year 2000 [19,20], which was used for tip-enhanced Raman spectroscopy (TERS) measurements. Here, we review the system introduced in reference [19], which has been the basic experiment setup of many subsequent studies in ANSOM. The system was constructed on the basis of an AFM using a silver coated cantilever set on an inverted microscope system. The incident laser beam $(\mathrm{CW}$, $532 \mathrm{~nm}$ ) was expanded 20 times with a beam expander and an evanescent mask was inserted in the beam path at the conjugated position of the pupil plane of an objective lens to achieve an evanescent illumination. This configuration realized strong longitudinal field at the focused spot and reduced undesirable background signals caused by the transmission components (corresponding to $N A<1$ ). The sample immobilized on a cover slip was set on an inverted oil-immersion objective lens $(\times 60,1.4 N A, 250 \mu \mathrm{m}$ WD). The Raman signal was efficiently collected by the same objective lens, which was separated from the incident light, by a half or a dichroic mirror. The collected Raman signal was introduced into a spectrometer and detected by a liquid nitrogen-cooled CCD detector.

Another transmission mode setup, which was also used frequently, was introduced in 2003 [26]. The main differences from the previous setup were in the controlling method of the probe tip and in the detection system. A laser beam $(633 \mathrm{~nm})$ reflected by a dichroic beam splitter was focused on the sample surface by an objective $(N A=1.4)$. A metal tip was positioned near the focus of the beam by means of a sensitive shear-force feedback mechanism. Raman scattered light was collected with the same objective and detected through a narrow band pass filter followed by a single-photon counting avalanche photodiode. 

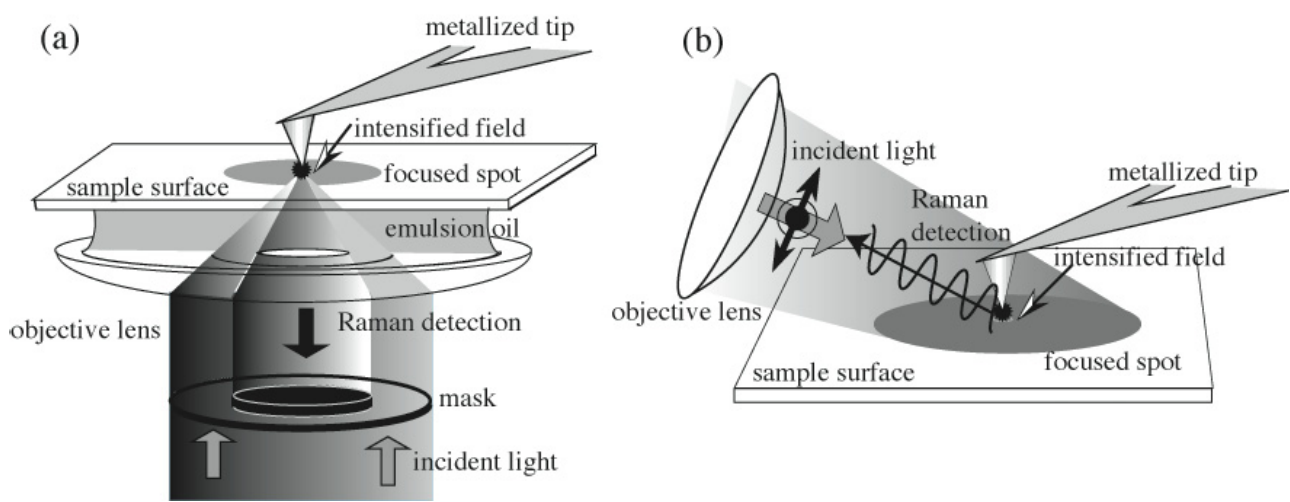

Fig. 2. Typical experimental setups of TERS: (a) transmission mode, (b) reflection mode.

\subsection{Reflection mode}

The reflection mode setup was first introduced by Anderson et al., which was still in an early stage of work in 2002 [27]. The instrument combined multimode AFM and a near-infrared Raman microscope $(785 \mathrm{~nm})$. The modified sample stage for rough positioning allowed AFM operation under Raman microscope objective $(\times 10)$ and realized side illumination of the tip-sample interface. They reported the point SERS spectra of diamond particles.

Later in 2003, Sun and Shen introduced a reflection mode TERS system [28], where they employed a long bent cantilever, which was made from tungsten wire through electrochemical etching and then coated with silver. This system was integrated on the platform of commercial nearfield system (AFM combined with micro Raman spectrometer). The compact design allowed the scanning head under the microscope objective $(\times 50,0.45 \mathrm{NA}, 13.8 \mathrm{~mm}$ WD). An argon-ion laser (514 nm) was focused onto the metal tip from the top of the cantilever. They reported a one dimensional TERS scanning of a silicon grating. In 2005, Mehtani et al. constructed a side illumination system [29]. This nearly horizontal confocal microscope used a long working distance (LWD) objective $(\times 50,0.42 \mathrm{NA}$, $20.5 \mathrm{~mm}$ WD) to facilitate the optical coupling of the Raman spectrometer with AFM stage. The objective lens was fixed on an $x-y$ stage controlled by step motors with an accuracy of $\sim 40 \mathrm{~nm}$ to position the objective with respect to the tip. The $514 \mathrm{~nm}$ line of an argon-ion laser was used. TERS signal was observed for methylene blue, PEDO/PPS polymer, CdS and carbon nanotubes at arbitrary positions of the film. Enhancement factor of $10^{3}-10^{4}$ was achieved with both silver- and gold-coated silicon nitride tips.

The most recent reflection mode experimental setup was introduced in 2006 [30]. An incident CW laser (532 $\mathrm{nm})$ was directed to the optical set-up using a single mode optical fiber. From the fiber output, the beam diameter of the laser was expanded $(\sim 10 \mathrm{~mm})$, and the polarization of the beam was set parallel to the tip axis, which corresponds to the $p$-polarization. This polarized light was focused on the sample using a LWD objective lens $(\times 20,0.28 \mathrm{NA}$, WD $30.5 \mathrm{~mm})$. The apex of the tip was adjusted onto the focused spot using the same principle as a contact-mode operation of AFM. The same LWD objective lens collected the scattering signal. A dichroic mirror directed the Raman signal output via a multimode optical fiber to a spectrometer. The spectrum was detected using a liquid nitrogen-cooled CCD camera. This setup also allowed for reflection-mode TERS imaging by means of scanning the stage.

In order to allow better access of the objective lens to the tip apex in reflection mode TERS, the images are obtained at the costs of the collection efficiency of Raman signal and the AFM performance. However, due to the advantage of measurement of opaque samples, this technique still has significant market for applications in semiconductor industries. Recently, reflection-mode TERS was successfully applied to nanoscale characterization of strained silicon $(\varepsilon-\mathrm{Si})$. This non-destructive characterization technique with a nano spatial resolution is essential for fabricating high performance electric devices using $\varepsilon$-Si substrates [30-32].

\section{Field enhancement by antenna structures}

As mentioned in the section of the experimental setup, the near-field probe acts as a nano-light source just like an antenna, which enhances the optical emission rate and controls the emission directions [33]. The investigation of the role of antenna structure to the incident light and the origin of the field enhancement is useful in designing the ANSOM probes. In this section we report some promising effects involve in ANSOM using plasmonic nanoprobe such as lighting rod, gap mode and image dipole effect.

\subsection{Amplification and controlling the propagation direction by an antenna}

The shape of the probes is often expressed as a sharp cone. On the apex of the probe, the density of electric field lines are so high leading to a tremendous field enhancement, the so-called "lightning rod effect" [34]. In TERS technique, the very weak signal detected by Raman scattering from a small number of molecules should be significantly enhanced to the level that can be detectable from farfield. The lightning rod effect is an electromagnetic field 
enhancement due to structural properties therefore universally applicable to any materials. This enhancement comes from the structure of the probe, which works in addition to other enhancement mechanism, such as plasmonic enhancement by metal free electrons [6] and chemical enhancement in SERS [21].

The role of antenna is not only to amplify the field but also to control the propagation directions. From this point of view, Taminiau et al. [35] experimentally demonstrated control of fluorescence emission direction of individual molecules by reversible coupling to a near-field probe, which acts as an optical monopole antenna. They showed that the angular emission of the coupled system was determined by a dominant antenna feature, that was the antenna design, regardless of molecular orientation. This indicates the role of the plasmon mode in the emission process and provides a guideline about how to exploit the largely available of radio antennas to direct emission in nano-optical microscopy, spectroscopy, and light-emitting devices, including single-photon sources.

\subsection{Gap mode enhancement}

It is theoretically predicted that a strong electric field is induced in interstitial gaps between two nanoparticles, which is called "hot spot" [36]. Raman scattering from a molecule is considered to be greatly enhanced when the molecule is adsorbed near the interstitial site. Many experimental results on SERS from molecules on aggregated nanoparticles are interpreted on the basis of the hot spot mechanism.

Imura et al. observed field enhancement in interstitial gaps between nanoparticles by aperture NSOM technique [37]. They investigated Raman spectra and two-photon-induced photoluminescence (TPI-PL) at single dimeric and trimeric aggregates of gold nanospheres by employing Ti:Saphhhire laser $(\lambda=785 \mathrm{~nm})$. Figure 3a shows topography of gold nanoparticle aggregates prepared on a cover slip. Isolated dimeric and trimeric nanoparticles could be found. Gap distance in the dimer was estimated to be about $1 \sim 10 \mathrm{~nm}$ from the topography and SEM image. They observed very strong Raman signals from gold aggregates while there were no detectable Raman signals from the isolated nanoparticles or from the substrate. Raman scattering at dimer 1 and 2 are shown in Figures $3 \mathrm{~b}$ and $3 \mathrm{c}$. Observed spectra show that the electric field is enhanced when the incident field is parallel to the interparticle axis of the dimer and in the case of perpendicular polarizations, the effect is hardly detectable. The near-field TPI-PL images from gold dimmers are shown in Figure 3d that sensitively reflect electric field distributions. The near-field images revealed that the excitation probability is highly localized at the interstitial site of the particles. In Figure 3e, images constructed from the nearfield Raman band of at around $1600 \mathrm{~cm}^{-1}$ are shown. The images again depend strongly on direction of the incident polarization. These results indicate that localized electromagnetic field at the interstitial site plays essential roles in
SERS and the incident polarization direction is also critical for the field enhancement. Note that these polarization dependencies would be promising element for a nanometric optical switching device.

In another study, gap mode structures were artificially formed by lithography technique to quantitatively study the gap-mode enhancement. Metallic bowtie nanoantennas consisting of two opposing tip-to-tip Au triangles were fabricated with triangle length of $75 \mathrm{~nm}$ and gap ranging from $16-488 \mathrm{~nm}[38,39]$. For light polarized along the line between the two triangles, the plasmon scattering resonance first shifted to blue, and then to red, gradually becoming decoupled with increasing gap. On the other hand, perpendicularly polarized excitation shows little dependence upon gap size. The authors explained these effects with dipole-dipole interaction picture. When the incident light is polarized along the dimer axis, the charge separation creates an attractive force, lowering the plasmon energy. As the gap length increases, this effect diminishes and the plasmon energy approaches that of a single isolated particle. Then the entire structure arrays are considered as a correction of two-dimensional dipoles and the coupling mechanism of these dipole array causes the spectral shift appeared in the experimental result.

The gap structure may modify the spontaneous emission rates from the molecules enclosed in the space. Purcell showed in 1946 that the amount of power emitted by an electromagnetic source depends on its surrounding [40]. This well-known effect called "Purcell effect" modifies the spontaneous emission lifetime of the system by the factor $F_{p}$,

$$
F_{p}=3 Q \lambda_{c}^{3} / 4 \pi^{2} n^{3} V
$$

where $\lambda_{c}$ is the wavelength of a single cavity mode, $Q$ is the quality factor $\lambda_{c} / \Delta \lambda_{c}, n$ is refractive index, and $V$ is the effective mode volume. This equation contains parameters related to the cavity geometry as well as the incident wavelength of light. The effect of gap separation, particle shapes and antenna structure on radiation rates has been extensively studied through finite-difference time-domain (FDTD) calculation $[41,42]$. According to these report, by varying the particle shapes and gap sizes, it is possible to increase the radiative decay rate by three orders of magnitude while keeping quantum efficiency larger than $80 \%$ in the near-infrared regime.

The resonance condition of surface plasmon is also affected by the gap distance. Pettinger et al. reported [43] the dependence of TERS composed of discrete Raman lines and the intense Lorentzian-shaped background by varying tip-substrate gap in STM setup. Remarkable blue shift of the background, which means the energy shift of the localized surface plasmon resonance, was observed.

It was reported for carbon-60 that the gap-mode TERS signal could be enhanced by almost two orders of magnitude in comparison with SERS signal [44]. Also, a recently developed technique of controlling the tip-sample distance in the tapping-mode operation of AFM [45] experimentally confirmed signal enhancement by two order of magnitude in gap-mode TERS. 
Y. Saito and P. Verma: Imaging and spectroscopy through plasmonic nano-probe

(a)

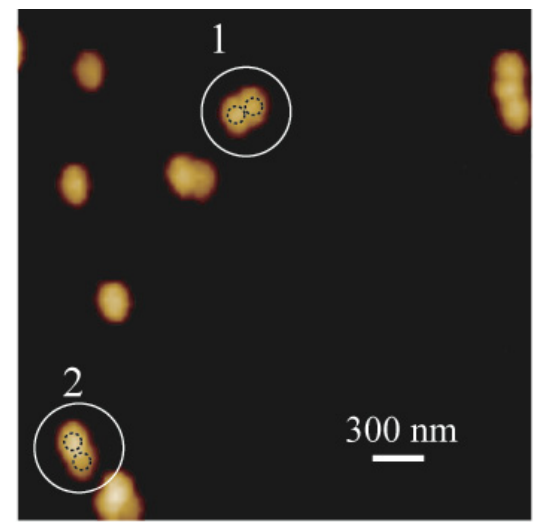

(d)

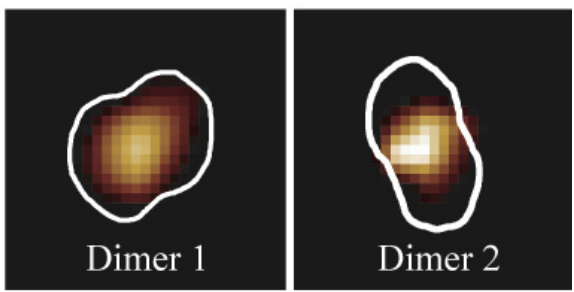

(b)

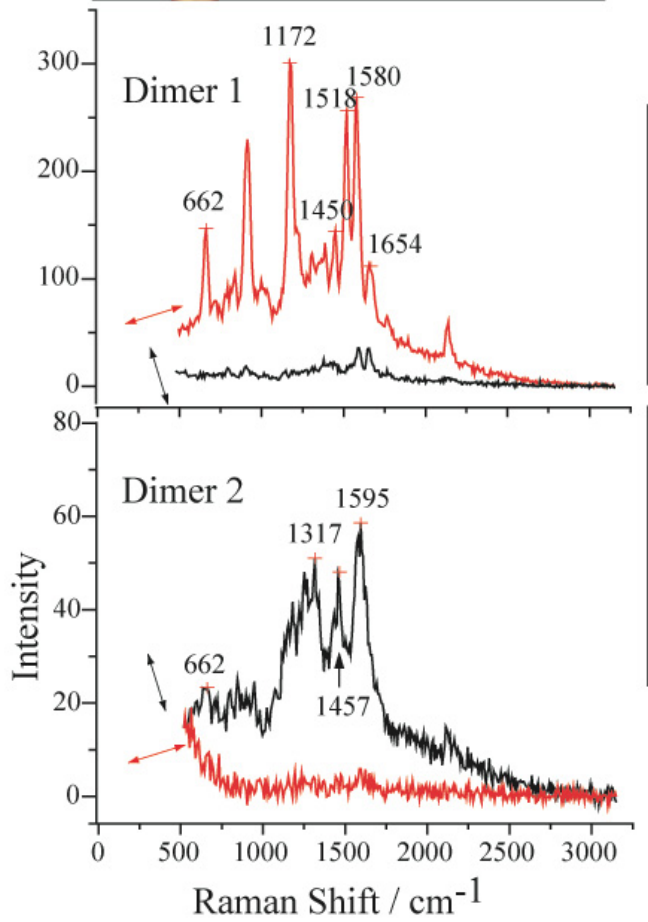

(e)
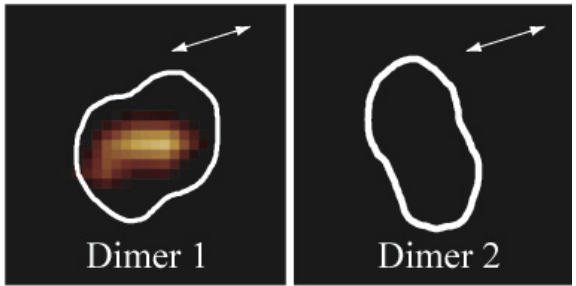

(c)
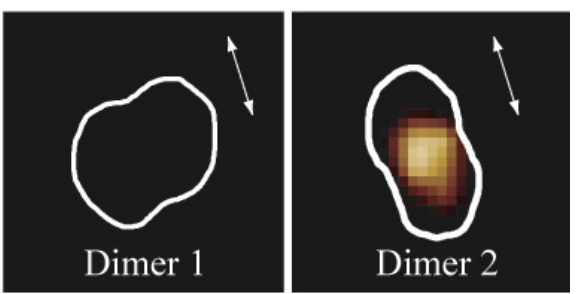

Fig. 3. (Color online) Gold nano-particle induced gap-mode enhancement excited by $785 \mathrm{~nm}$. (a) Topography of aggregated and isolated gold nanoparticles. (b) and (c) Near-field Raman spectra at dimer 1 and 2 respectively, taken at two different incident polarization directions shown by arrows. (d) Near-field TPI-PL image of dimer 1 and dimer 2 (e) near-field Raman images at dimer 1 and 2 obtained for bands near $1600 \mathrm{~cm}^{-1}$. The images were measured at different incident polarization directions indicated. No analyzer was placed at detection pass. Image sizes are $540 \mathrm{~nm} \times 540 \mathrm{~nm}$. Permission to reprint is granted by the American Chemical Society.

Fabricating a gap-mode structure on a near-field probe apex may be fascinating for strong enhancement and signal emission control, however at this stage it is not easy to control the gap distance by $1 \mathrm{~nm}$ accuracy. Neither FIB milling nor self-assembled chemical method can fabricate such a gap-mode probe that is stable enough to use. The future technologies for easy and precise fabrication are expected.

\subsection{Image dipole effect}

In standard experimental configurations of ANSOM discussed earlier, one can easily create a gap structure between the substrate and the probe apex. Considering the interaction between the near-field probe and the substrates, the enhancement efficiencies are expected to modify by the material combinations. For example, the combination between silver tip - silver substrate will give a strong electric field in the gap while silver tip - glass substrate will give only a week enhancement. This property to produce an optical contrast is useful in scattering type ANSOM in infrared imaging [46] and refractive index imaging [47]. If the tip apex is approximated as a single dipole, the electric field distribution around the tip apex can be written as follows:

$$
\begin{aligned}
& E_{\text {dipole }}= \\
& \qquad \cos \theta\left(\frac{2}{r^{3}}-\frac{2 i k}{r^{2}}\right) e_{\|}+\sin \theta\left(-\frac{1}{r^{3}}+\frac{i k}{r^{2}}+\frac{k^{2}}{r}\right) e_{\perp},
\end{aligned}
$$


where $e_{\|}$and $e_{\perp}$ are parallel and perpendicular unit vector to the dipole axis, $k$ is incident light propagating vector, and $r$ and $\theta$ represent the distance from the dipole center and the angle from the dipole axis, respectively. Estimated field enhancements between the tip and the substrate gaps can be written as a simple model know as an "image dipole effect" [48]. In this method, one can assume an opposite charge $-q_{\text {image }}$ at the inverse position inside the substrate which is the reflection of the real charge $q_{\text {real }}$. Accordingly, the image dipole creates electric field at the vacuum side of the plane as:

$$
q_{\text {image }}=\frac{\varepsilon-\varepsilon_{0}}{\varepsilon+\varepsilon_{0}} q_{\text {real }},
$$

here $\varepsilon$ and $\varepsilon_{0}$ are the dielectric constants of the media and the vacuum. The image dipole effect is constructive when the dipole is perpendicular to the interface and distractive when it is parallel to the interface. The resulting electric field in the gap is the summation of the real dipole and the image. This can also be used for effective background rejection in near-field experiments [49]. In this study, the authors systematically investigated the polarization dependent image dipole effects near a flat gold surface. A gold-nanoparticle-functionalized tip was employed as a local field scatterer and they applied either parallel or perpendicular polarization to the tip axis to separate the dipole contributions from the signal.

Even when we do not utilize a metallized probe, the image dipole effect contributes the near-field enhancement considerably caused by the multiple image dipole interaction between the sample/substrate and probes [50].

\section{Polarization properties in near-field experiments}

Polarization measurements provide unique structural information from which one can extract a wealth of knowledge on the orientation, inter-molecular interactions and symmetry deformations of molecules. Polarizationcontrolled ANSOM is a powerful tool for surface characterization, since it yields information about both chemical composition and assembled structures. Unlike conventional Raman spectroscopy, the polarization direction in ANSOM is characterized by two axes, parallel ( $p$-polarization) and perpendicular ( $s$-polarization) directions to the probe tip axis. In this section, we will review the polarization treatments, which is unique to near-field.

\subsection{Polarization measurement under a high $N A$ objective lens}

When a high $N A$ objective lens tightly focuses a linearly polarized light, the resulting polarization at the focal plane consists of both $p$ and $s$-components [51,52]. Besides this polarization admixture, the field intensity distributions of each component cause a problem when the spatial resolution is down to nanometer scale. Figure 4 shows the
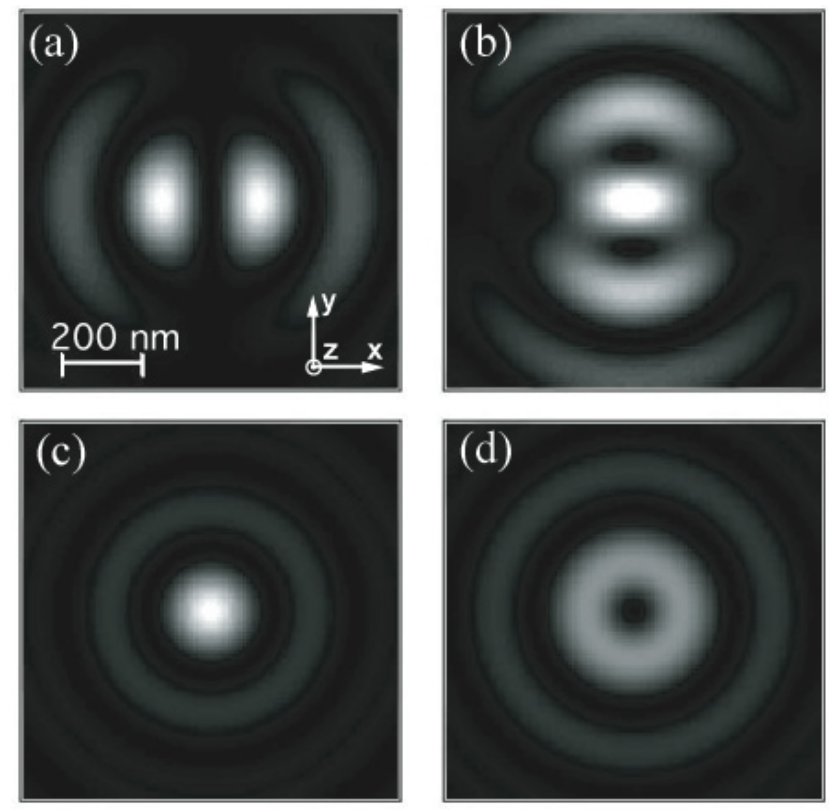

Fig. 4. Calculated field intensity distribution at a focused laser spot: (a) z-polarization component created by linearpolarization ( $x$-direction) (b) $x$ and $y$ polarization component (c) $z$-polarization component excited by radial polarization (d) $x$ and $y$ polarization component excited by azimuthal polarization.

calculated field intensity distribution of a linearly polarized ( $x$-direction) light at the focal plane tightly focused by an objective lens $(N A=1.4)$, (a) for $p$-polarization and (b) for $s$-polarization. The electric field of $p$-polarization is canceled at the center of the spot, which creates alternately two side peaks as appeared in Figure 4a. In ANSOM measurements, one needs to carefully adjust the tip onto one of the two $p$-polarization field components. On the other hand, field intensity of $s$-polarization shows a maximum at the center as seen in Figure $4 \mathrm{~b}$.

By controlling the incident polarizations, we can choose the polarization condition at the focal plane either $p$ or $s$. Radially or azimuthally distributed polarizations form perfect $p$ or $s$-polarization. Figures $4 c$ and $4 \mathrm{~d}$ show the calculated total electric field intensity distributions at a tightly focused spot using radial $(p-)$ and azimuthal $(s-)$ polarization. In the $p$-polarization configuration, the electric field intensity in the longitudinal direction (along the tip axis) is the strongest at the center as in Figure 4c. In the $s$-polarization configuration, the lateral field intensity is the strongest on the circumference as in Figure 4 d. Note that both $x$-polarization dominant and $y$-polarization dominant areas are formed in the case of the $s$-polarization configuration.

We also have to consider that the signal collection efficiencies for the $p$ and $s$-polarization directions in the backscattering configuration are different. Assuming that the tip apex is approximated as a single dipole, the detection efficiencies of $s$ and $p$-polarizations are indicated by factors $A$ and $B$, respectively, which are shown below as 
functions of the objective lens cone angle $\theta_{m}[53]$

$$
\begin{aligned}
& A=\pi^{2}\left(\frac{4}{3}-\cos \theta_{m}-\frac{1}{3} \cos ^{3} \theta_{m}\right), \\
& B=\pi\left(\frac{2}{3}-\cos \theta_{m}+\frac{1}{3} \cos ^{3} \theta_{m}\right) .
\end{aligned}
$$

A simple estimation tells us that the factor $B$ is always less than $10 \%$ of $A$ which means the detection efficiency of $p$-polarization is much lower than that of $s$-polarization [54]. Also, one needs to consider the emission patterns of each dipole component. This property was visualized by Moerland et al. [55] that the fluorescence emission patterns could clearly be distinguished by raster scanning the near-field probe on the fluorescence molecules. The molecules oriented to $z$ direction perpendicular to the sample surface show clear doughnut pattern of radiation while $x-y$ oriented molecules only show round intensity pattern. By employing the polarizing beam splitter and two APD detectors, they obtained two detectable polarization components simultaneously.

\subsection{Metallized tips and polarizations}

The polarization efficiency in ANSOM, especially in TERS, strongly depends on the shape of a metallized tip. A $p$-polarized plane wave illumination onto the conically shaped tip can efficiently induce tip-enhancement while the $s$-polarized light cannot. It is generally accepted that tip-enhancements requires $p$-polarized component in the incident light $[26,50]$. However, the enhancement of Raman signal also depends on the direction of transition moment of molecules. Some vibrational modes couple well with $s$-polarization than $p$. For example to excite the Raman mode from the $G$-band of carbon nanotubes, $s$-polarization component is required [56].

Metallized tips prepared by variety of ways may have different shapes like nanospheres or sharp conical shapes [22,57]. A single nanosphere with size much smaller than the wavelength can be considered as a dipole when light field is incident on the sphere [58]. This dipole would generate electric field perpendicular as well as parallel to the incident field. If we approximate the tip apex as a sphere, the electric field distribution can be calculated by employing prolate spheroidal coordinate [59]. The calculated field distribution formed around a sphere shows that the amplitude of the $s$-polarization field is just half of that of the $p$-polarization field [22]. Compared to the sphere case, when the tip apex is sufficiently sharp (the ratio between the long and the short axes becomes much larger than 1), only negligible field is formed by $s$-polarized excitation while $p$-polarized excitation shows the strong field concentration underneath the tip. This indicates that only $p$-polarization is used for field enhancement using a sharp metallic tip.

The polarization property affected by the near-field probe is more serious in the case of apertured probes. It may couple with the sample polarization and amplify or scramble the sample polarizations. In the case of apertured probes, the shapes of the probes are directly related to the polarization on the sample plane. For example, a triangular nano-aperture employed in fluorescence imaging, can determine the orientation of each fluorescent molecules [60]. One needs to design the near-field probe suitable for the desired polarization components.

\subsection{Depolarization effect}

In reflection mode illumination which is summarized in Section 2, the polarization configuration can be easily controlled to either $p$ or $s$-polarization. However, the far-field background signal in ANSOM may cause a serious problem, especially in reflection mode, due to large focus spot associate with an objective lens that has a long working distance. Also, for a thin-layered sample grown on a bulk, signal from bulk area interferes with the signal from the thin sample layer. In such cases, a background reduction can be achieved by exploiting the local light depolarization induced by the metallic tips. Recent experimental studies show that the optical response of the tip causes the $s$-to-p-polarization conversion and vice versa which is called "depolarization effect". Both dielectric and metalcoated tips have been investigated and depolarization effect up to $5 \sim 30 \%$ have been measured for various tip shapes and polarization directions [61]. Raman scattering from crystals can in fact be strongly polarized because of symmetry-related selection rules. The Raman signal originating from the tip scattering can therefore be selectively isolated by analyzing the cross-polarized Raman scattering. An extensive study to determine the tip interaction with polarized light in TERS has been recently published [62].

Here we discuss one such experimental demonstration of the depolarization experiments for TERS sensitivity improvement [63]. In this paper, authors reduced the far-field signal by controlling the polarization state of the incident and scattered Raman as well as by manipulating the polarization of a crystalline sample, which strongly depends on the polarization and propagation of the incident light. The sample was a $30 \mathrm{~nm}$ layer of $\varepsilon$-Si grown on a SiGe substrate. TERS measurements in reflection mode were carried out using $\mathrm{Si}_{3} \mathrm{~N}_{4}$ tip, with and without a silver coating in both parallel polarization (incident; $p$-polarization, analyzer; $p$-polarization) and cross polarized ( $s-p)$ configurations. The results are shown in Figure 5. The sample azimuth was $45^{\circ}$, as determined from far-field experiments to achieve the largest signal for analyzer placed in parallel configuration. In the parallel polarization condition, as shown in Figure 5a, the far-field Raman signals from the LO phonon mode of Si can efficiently pass through the analyzer. The decrease in Raman intensity of SiGe when the silver-coated tip was in contact could be attribute to the depolarization effect and incident intensity loss caused by the shadowing effect in the tip. The enhancement is quantified in the table normalized by SiGe peak intensity in farfield. In cross polarization conditions, as indicated in Figure $5 \mathrm{~b}$, on the other hand, the tip enhancement was very 
(a)

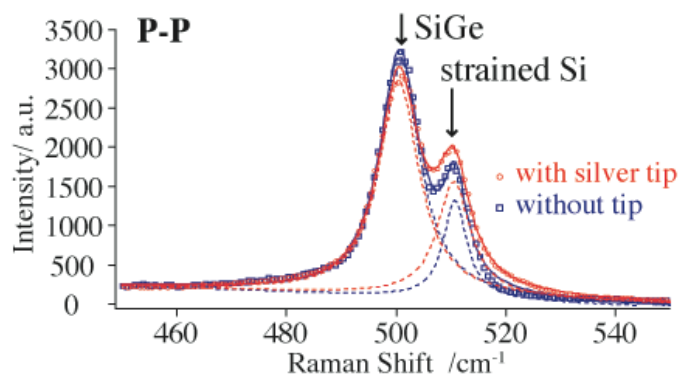

\begin{tabular}{c|c|c}
$\mathbf{P}-\mathbf{P}$ & SiGe peak & Strained Si peak \\
\hline Tip-Enhancement & $1 \times$ & $1.84 \times$ \\
\hline $\begin{array}{c}\text { Depolarization } \\
\text { effect }\end{array}$ & $0.89 \times$ & $0.89 \times$
\end{tabular}

(b)

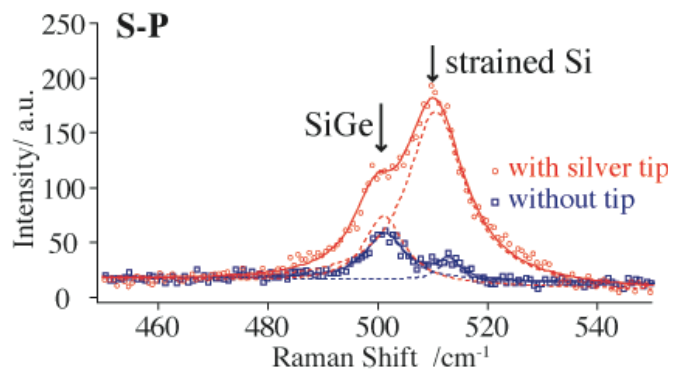

\begin{tabular}{c|c|c} 
S-P & SiGe peak & Strained Si peak \\
\hline Tip-Enhancement & $1 \times$ & $11.7 \times$ \\
\hline $\begin{array}{c}\text { Depolarization } \\
\text { effect }\end{array}$ & $1.34 \times$ & $1.34 \times$
\end{tabular}

Fig. 5. (Color online) TERS spectra of strained silicon prepared on SiGe substrate obtained by 442 nm excitation with objective lens $0.28 N A 20 \times$ in reflection mode configuration, $s$-polarization illumination and $p$-polarization detection $(s-p)$ configuration, the sample azimuth was fixed at the angle which shows maximum far-field intensity at $p$ - $p$ configuration. The table shows the enhancement efficiency of each polarization configurations. Figure provided courtesy of Motohashi.

large, compared to parallel polarization configuration, despite the $s$-polarized incident and $p$-polarized scattering detections. In this case, tip enhancement is expected to occur only on the Raman signal at the detection. These results strongly suggest that the combination of polarizer, analyzer, and sample azimuth that gives minimum farfield background signal can be utilized in the TERS experiment to obtain high-contrast near-field Raman signal.

\section{Resonance wavelength of surface plasmon}

Besides extremely high spatial resolutions, plasmonic nano-imaging provides variety of material information combined with spectroscopic techniques such as Raman scattering, infrared absorption, fluorescence emission, $\mathrm{THz}$ spectroscopy, etc. The information one can obtain would depend on the wavelength of light one is using. For example, Raman spectroscopy employing visible wavelength is to excite inelastic scattering by molecular vibrations. On the other hand, infrared absorption can excite similar states but with different selection rules by directly targeting the vibrational energy level of the molecule using infrared light. If the light wavelength is in resonance with or beyond the electric energy level of the samples usually visible of near-UV regions, we can expect the light emission reflecting the electric state nature of the sample molecules. $\mathrm{THz}$ spectroscopy is used for observation of much lower energy phenomena, e.g., identification of unique rotational and intermolecular-vibrational responses of materials that falls within this frequency range.

\subsection{Surface plasmon tuning in visible wavelength}

Near-field experiments have mostly been developed and operated in the visible wavelength region [9-20]. In this wavelength range, ANSOM techniques combined with Raman and fluorescence spectroscopy are commonly used. In terms of tip enhancement, the spectral match between excitation laser and the resonance frequency of the tip is required. It is known that the resonance of surface plasmon in a metal nanostructures is sensitive to the optical constants of its surrounding materials. Therefore, tuning of the resonance properties of a metal-coated tip by changing the accessible parameters such as coating materials or the shapes seems realistic than to change the excitation laser source.

Here, we briefly summarize the coating effect of the tip by simple electromagnetic formulations. Assuming that the tip apex is a sphere much smaller than the excitation wavelength, the quasi-static model is feasible. The polarizability $\alpha$ of the tip can be given by:

$$
\alpha=\frac{4 \pi}{3} \varepsilon_{0} R^{3} \frac{(\varepsilon-1)}{(\varepsilon+2)},
$$

here $\varepsilon_{0}$ and $\varepsilon$ denote the vacuum and relative permittivity of the material as the function of wavelength $\omega$, and $R$ represents the radius of the tip apex [64]. In the case of a coated sphere with the core diameter $R$ and the thickness of the coating $d$, the static polarizability can be given by modifying equation (5) as:

$$
\begin{aligned}
\alpha=\frac{4 \pi}{3} & \varepsilon_{0}(R+d)^{3} \\
& \times \frac{\left(\varepsilon_{s}-1\right)\left(\varepsilon_{c}+2 \varepsilon_{s}\right)+\left(\frac{R}{R+d}\right)^{3}\left(\varepsilon_{c}-\varepsilon_{s}\right)\left(1+2 \varepsilon_{s}\right)}{\left(\varepsilon_{s}+2\right)\left(\varepsilon_{c}+2 \varepsilon_{s}\right)+\left(\frac{R}{R+d}\right)^{3}\left(\varepsilon_{c}-\varepsilon_{s}\right)\left(2 \varepsilon_{s}-2\right)},
\end{aligned}
$$

here, $\varepsilon_{s}$ and $\varepsilon_{c}$ denote the permittivity of the shell and the core materials, respectively, and the refractive index 


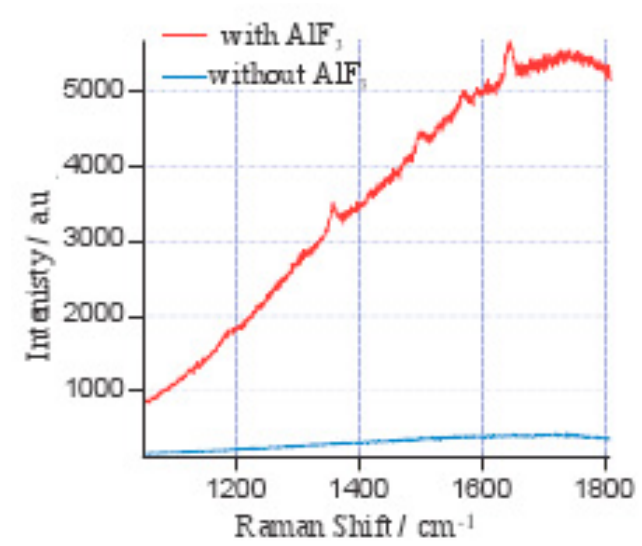

Fig. 6. (Color online) SERS spectra of rhodamine $6 \mathrm{G}$ obtained by $488 \mathrm{~nm}$ excitation, objective lens $0.6 \mathrm{NA} \times 40$, $20 \mathrm{~nm}$ silver substrates were prepared by vacuum evaporation either on silicon (blue line) or $\mathrm{AlF}_{3}$ (red line) substrate.

of the surrounding is set to 1 . By substituting $\varepsilon_{s}=1$, one can confirm that equation (6) reduces to equation (5). The resonance condition is achieved when the denominator approaches zero, and hence we can modify the resonance frequency by changing the refractive index of the combination of the core and the shell material.

Recently, Ag coated dielectric tips with different refractive indices of substrates were studied for Raman enhancement efficiencies $[65,66]$. Following the idea of plasmon resonance tuning, the performance of the Ag coated tip has been significantly improved by using a low refractive index AFM tip. The experiment was targeted for $488 \mathrm{~nm}$ illumination with commercial AFM tips. The optical properties of AFM tips can influence the resonance properties of the $\mathrm{Ag}$ coating, such as the resonance frequency, the resonance width and the associated field enhancement, which was systematically investigate by numerical analysis (finite element method; FDTD). Due to the geometry of the tip, one has to consider several plasmon modes on the tip apex that make the resonance structure more complex compared to the case of a spherical particle. One of the most prominent features of the relationship between the refractive index and the resonance wavelength is that the resonance modes are red shifted with increasing refractive index of the AFM tip. Figure 6 shows the experimental example of plasmon resonance tuned by refractive index modification of the tip. The tipcoating process for these experiments was similar to the one discussed in reference [66]. The sample was rhodamine $6 \mathrm{G}\left(10^{-7} \mathrm{M}\right)$ spin coated on $20 \mathrm{~nm}$ thick $\mathrm{Ag}$ surfaces. The substrates were either $\mathrm{AlF}_{3}(n=1.38)$ coated or uncoated on silicon $(n=4.2)$. The red line indicate the SERS spectrum on the $\mathrm{AlF}_{3}$ substrate and blue line indicate the result on the silicon substrate. The efficiency of plasmon resonance is dramatically modified by the tuning the refractive index of the base media. In the case of $\mathrm{AlF}_{3}$, the calculation shows the plasmon resonance peak is expected to appear around $500 \mathrm{~nm}$, which qualitatively explains strong enhancement by $488 \mathrm{~nm}$ laser excitation [65].
Coating material by vacuum evaporation is not the only way to modify the refractive index of the tip. In one of such examples [67], silicon tip was oxidized to $\mathrm{SiO}_{2}$ by heating the tip at $1000{ }^{\circ} \mathrm{C}$ under water vapor atmosphere. What is interesting here is that the shape of the tip apex still keeps its original sharpness even when the oxide layer becomes as much as $100 \mathrm{~nm}$.

Another important point for the wavelength tuning is to control the metal particle shapes [68-70]. If the particle shape is not spherical, it may have several plasmon resonance modes [65]. Luminescence from individual gold nanorods with different shapes was investigated by Bouhelier et al. [71]. Two photon excited gold nanoparticles shows light emission originating from the radiative decay of surface plasmons. They also measured a blue shift of the photoluminescence peak wavelength with decreasing aspect ratio of the nanorods as well as an optically tunable shape dependent spectrum of the photoluminescence. There are varieties of works related to the formation of metal nanoparticles and its relation to surface plasmon, however, fixing these particles on the near-field probe apex for scanning is another big issue.

\subsection{Surface enhanced infrared absorption}

Surface enhanced infrared absorption (SEIRA) is a complementary vibrational spectroscopic method to SERS, which can be performed by direct IR excitation of molecules on metal structures [72-74]. As the surface plasmon on metal substrate enhances the electric field of incident light and scattered Raman signals in SERS process, an increased IR absorption by adsorbed molecules are observed in SEIRA when the plasmon resonance is shifted to mid IR. As mentioned earlier, the refractive index control is significantly effective for plasmon resonance tunings, nano shell structure, e.g., silica core coated with metal provides a remarkable high-quality substrate for SEIRA spectroscopy with plasmon resonances turned to the infrared region of the spectrum. In SEIRA, vibrational modes of molecules with a change of dipole moment perpendicular to the substrate are enhanced and therefore this technique has potential for providing the information about orientation of adsorbed species with respect to the surface. Since its invention, SEIRA has been used in numerous applications in chemical analysis and characterization and biochemical sensing [75].

Aggregated nano shell structures are useful and SEIRA spectra with strong absorption enhancement across the entire spectral range of the chemical fingerprint region of $700 \sim 3300 \mathrm{~cm}^{-1}$ can be obtained [76]. Based on a statistical evaluation of aggregate geometries combined with FDTD modeling of the infrared hot spots characteristic of the constituent structures, the enhancement factor for SEIRA from para-mercaptoaniline molecules is estimated to be of the order of $10^{4}$. The sample was dispersed on nanoshell aggregates and examined in transmission configuration by FT-IR commercial spectrometer with MCT detector. 
Another example in the IR wavelength range is scattering NSOM ( $s$-NSOM). This method and succeeding $\mathrm{THz}$ spectroscopy do not utilize the plasmon enhancement. Huber et al. [77] and Brehm et al. [78] demonstrated that $s$-NSOM could determine infrared fingerprint spectra of individual poly-methyl methacrylate nanobeads and tobacco mosaic virus (TMV) as small as $18 \mathrm{~nm}$. Both materials possess distinct vibrational resonances in the wavelength region near $6 \mu \mathrm{m}$ covered by a line-tunable CO laser. The authors used commercial Pt-covered Si cantilever tip with tapping mode and the interferometric technique, together with second harmonic signal demodulation. The local optical response measured by elastic light scattering from the tip relates to the complex dielectric value of the sample. The spectroscopic $s$-NSOM can assess the optical dispersion of the sample from which we can recognize the infrared fingerprint signature and finally assign the chemical composition. Amplitude and phase spectra were found surprisingly strong even at a probed volume of only $10^{-20}$ litter, and robust in regard to particle size and substrate. Though the understanding of the spectra is still under discussions, this method makes infrared spectroscopic $s$-NSOM versatile tool for chemical and protein-secondary structure identification.

\subsection{Near-field experiments in $\mathrm{THz}$ region}

Besides many attempts to access the near-infrared and mid-infrared ranges in order to image the vibrational resonances of a specimen, further development towards $\mathrm{THz}$ wavelength is promising, for mapping of biological cells and for semiconductor device inspection, where spatial resolutions is necessary for tracing the distributions of materials $[79,80]$. In the case of $\mathrm{THz}$ imaging where the spatial resolution is limited by the wavelength, for example $300 \mu \mathrm{m}$ for $1 \mathrm{THz}$, near-field microscopy is just the suitable technique to obtain sub-wavelength resolution in this spectroscopy. Recently, $\mathrm{THz}$ imaging microscopy ranging from $0.2-40 \mathrm{THz}$ was realized with resolution better than $\lambda / 10[81,82]$.

The principle for $\mathrm{THz}$ NSOM is based on Mie scattering that probes the near-field by diffuse scattering. By considering the polarizability $\alpha$ of the tip given in equation (5), Mie theory leads to cross-section of the scattering efficiency $C_{s c a}$ and the absorption $C_{a b s}$ as:

$$
C_{s c a}=\frac{8 \pi^{3}}{3 \lambda^{4}}|\alpha|^{2}, \quad C_{a b s}=\frac{2 \pi}{\lambda} \operatorname{Im}\{\alpha\} .
$$

The cross-sections for scattering and absorption scale down with $1 / \lambda^{4}$ and $1 / \lambda$, respectively, which is extremely small in the far infrared [79]. Thus further improvement of $\mathrm{THz}$ near-field imaging is necessary for the practical application of this wavelength range. Configurational resonances like antenna system are expected to occur in $\mathrm{THz}$ near-field imaging. Lock-in detection at the modulation frequency also plays a dominant role in other spectral ranges such as the near infrared. Theoretical prediction is also important to estimate the behavior of the near-field light and the scanning probe [83]. The spatial resolution of apertureless $\mathrm{THz}$ imaging was reported to be $150 \mathrm{~nm}$ for $2 \mathrm{THz}$ pulses by Kersting et al. [84].

We note here that there are reports related to SERS effect in UV wavelength region [85], however to the best of our knowledge, application of UV wavelength to the tipenhancement and nano-imaging has not been reported so far. The diffraction limit of UV lights itself is down to $100 \mathrm{~nm}$ without a near-field probe. It has been reported that a compact capillary discharge UV laser can realize an imaging with a spatial resolution of 120-150 nm [86,87].

\section{Applications and advanced measurements}

\subsection{Biological application of ANSOM}

In recent years, several applications of ANSOM, especially Raman spectroscopy (TERS), have been reported by many groups in bioscience [88]. Since the tip-enhancement technique can provide resolutions compared to the size of protein and other biological entities (about $10 \sim$ $30 \mathrm{~nm}$ ) [6], this method seems to be a promising tool for biological analysis, though there are some problems in employing it to protein or lipid based biomaterials. In most cases, Raman signals generated from biological samples are not strong enough as inorganic substances or carbon materials. Furthermore, the sample itself can hardly stand for the long time exposure of laser illumination, therefore, bleaching and photo damage of the sample may be big problems. Another concern is strong Raman signals from carbon contaminations that may originally exist in the sample or may come from the molecular decomposition products. In some cases, samples should be placed in water, since many of the biological systems need wet conditions to preserve their original functions. This means that the ANSOM setup should be able to operate in water, which is a challenging alteration from the basic ANSOM setup in air [89-91]. Taking these issues into account, the works introduced below demonstrate that ANSOM has the potentiality to collect detailed description of large biomolecules.

Koopman et al. demonstrated liquid-NSOM using a diving bell concept [89]. Individual fluorescent molecules on the membranes of cells in solution were imaged with a spatial resolution of $90 \mathrm{~nm}$. They directly visualized nanometric size domain of the C-type lectin on the membrane of dendritic cells. Another example is the cytchrome C studied by TERS [92]. By the virtue of its sensitivity and superior resolution, TERS successfully detected both the heme and amino acid vibrational bands of cytchrome C using resonance excitation at $532 \mathrm{~nm}$. In another study [93], TERS spectra of DNA pyrimidine bases were measured and compared with standard SERS and Raman measurements of the nucleotides and the pure bases. The results were discussed in terms of electromagnetic and chemical effects of SERS enhancement of the probe.

The softness of the biological sample and observation of only a small number of molecules sometimes cause the 

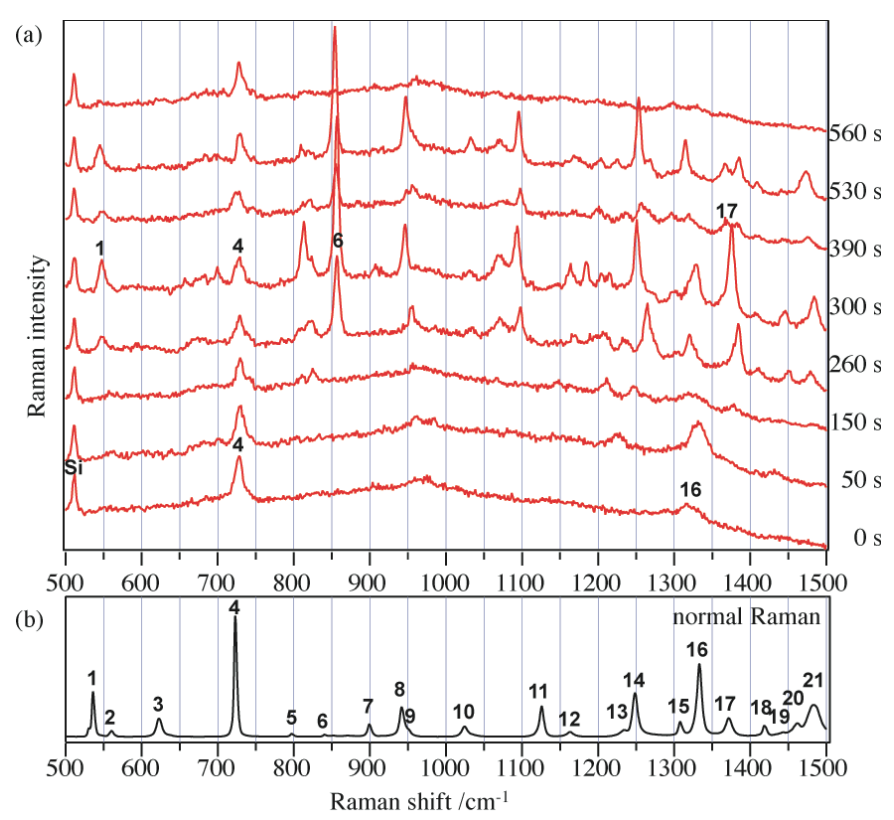

Fig. 7. (Color online) TERS spectra of adenine nano crystals: (a) selected 8 characteristic spectra obtained at different times, (b) normal Raman spectra of bulk adenine for comparison. Figure provided courtesy of Ichimura.

fluctuation within few seconds in time. The temporal fluctuation of spectral shapes in TERS of DNA molecules has been reported [94]. The TERS spectra of nanocrystalline cluster of adenine molecules showed distinct temporal change including fluctuation of peak frequencies and intensities and extraordinary enhancements of several peaks. Time-dependent characteristic spectra are shown in Figure 7a for facile comparison of their spectral shapes. These TERS spectra were also compared with a normal Raman scattering spectrum, shown in Figure $7 \mathrm{~b}$ of a bulk adenine sample with no tip attachment. For simplicity of reference, the vibrational modes in Figure $7 \mathrm{~b}$ are sequentially numbered. A stable silicon peak originating from the tip material was observed in all spectra and hence could be used as a reference for estimating the fluctuations. For spectra recorded during the time $0-50 \mathrm{~s}$, only two peaks numbered 4 and 16, were observable. At $50 \mathrm{~s}$, some new peaks started to appear, the frequency of which agreed with the adenine peak observed in Figure $7 \mathrm{~b}$. In the time region 260-300 s, many new peaks emerged. Most peaks fluctuate during the entire experimental period. The fluctuating TERS signals originate primarily from the molecules in the first layer of the $5 \mathrm{~nm}$ thick adenine nanocrystalline clusters. The possible origin of the temporal changes was attribute to the changes of molecular orientation under the metal-coated tip, which is responsible for changing the adsorption angle and the angle of local incident polarization for the molecules adsorbed to the tip. This result suggests the importance of sampletip interaction at molecular level in the interpretation of TERS spectra.

Besides biological samples, the spatial resolution and the sensitivity of ANSOM has also been applied for variety of samples, e.g., strained silicon that was discussed earlier [31-33], InAs quantum dots [95], GaN thin layers [96], or block-copolymer nanostructures [97]. These samples are basically connected to industrial products indicating that the ANSOM can be a practical tool to examine and evaluate microelectric devices in near future.

\subsection{Single molecule detection}

It can be said that the ultimate resolution goal for NSOM is single molecule observation. Ensemble average from large number of molecules gives limited information of the phenomena, for example, the understanding of the diversity of biological process requires methods that can address a single protein in their natural environment and provide insights into structural and functional properties as well as the local distribution of each protein. So, to avoid the statistical averaging in space and time, single molecule observation is necessary. At this stage, not many molecules can be targets because of the lack of the sensitivity. Sometimes it is hard to distinguish whether or not the observed signal is coming from only one molecule. One possible evidence to present the single molecule sensitivity is related to sample preparation by using extremely diluted sample to ensure that only a single molecule is expected within the detection volume. Another evidence is spectroscopic fluctuations, so-called "blinking" phenomena, including intensity and frequency shifts [98].

Due to the weak cross-section of other optical processes, single molecule experiments are usually demonstrated through photoluminescence detections [99]. In this case, one has to consider the fact that emission lifetime may be affected by the presence of a metal tip [100-102]. The research group of Sandoghdar investigated the coupling of a single spherical gold nanoparticle acting as a nanoantenna [103]. They positioned a metallic particle close to the sample molecules and observed a shortening of the lifetime by 20 -folds.

There are also reports on single molecule nano-probe detection in Raman spectroscopy. Zheng et al. presented single molecule resonance TERS from brilliant crystal blue (BCB) submonolayers adsorbed on a planar Au surface with Ag tips [104]. A gap of $1 \mathrm{~nm}$ between Ag tip and the Au substrate was employed to create a highly enhanced electric field and to generate Raman scattering from an area of $100 \mathrm{~nm}^{2}$.

\subsection{Novel methods in NSOM}

In this section, some of the novel measurements methods in near-field experiments such as pressure, temperature or electrons are briefly introduced. Pressure dependence Raman spectra were demonstrated by Verma et al. [42] and Yano et al. [105]. They imposed uniaxial pressure on carbon-60 and single walled carbon nanotube by the AFM tip, which showed spectral shifts due to the bandgap modification caused by the local stress. 
Intense multiphoton electron emission is observed from sharp metallic tips $(\sim 20 \mathrm{~nm}$ radius $)$ illuminated with femto-second light pulses. By monitoring the electron emissions, local field enhancement, evidence by current nonlinear light generation, confines the emission to the tip apex. Electrons are emitted from a highly excited nonequilibrium carrier distribution, resulting in a marked change of the absolute electron flux and its dependence on optical power with the tip bias voltage [106].

Temperature control is another issue in ANSOM experiments. Gerhardt et al. presented scanning near-field excitation spectra of single molecules embedded in a solid matrix [107]. The experimental setup consisted of a combined scanning confocal and near-field optical microscope that operated at $T=1.4 \mathrm{~K}$. By varying the tip-sample separation, they modified the line shape of the spectra, demonstrating the coherent nature of the interaction between the incident laser light and the excited state of the molecules. On the contrary, Wilde et al. and Chapuis et al. introduced thermal heating effect on ANSOM by employing a hot sample holder $[108,109]$. They described an infrared ANSOM that operated without any illumination, analogue of a night-vision camera making use of the thermal infrared evanescent emitted by the surface, and behaves as an optical scanning tunneling microscope. They showed the first images of thermally excited surface plasmon and demonstrated spatial coherence effects in nearfield thermal emission.

\subsection{Plasmonic superlens}

In previous sections, we have reviewed super resolution imaging method using a near-field probe. However, a completely different approach of plasmonic imaging to overcome the limitation of a conventional lens is presented by several groups recently. An unconventional alternative to a lens, a slab of negative refractive index materials has the power to focus all Fourier components of a 2D image, even those that do not propagate in a radiative manner. Such a lens has been named as plasmonic "Superlens" [110].

The wave vector components $k_{x}$, and $k_{y}$, representing the Fourier decomposition of an object, have larger values corresponding to finer structures and smaller values corresponding to larger structures. In conventional optics, $k_{z}$ takes only real values, which imposes a restriction on the wave vectors components $k_{x}$, and $k_{y}$ as:

$$
\frac{\omega^{2}}{c_{0}^{2}}=\frac{2 \pi}{\lambda}>k_{x}^{2}+k_{y}^{2} .
$$

Imaginary value of $k_{z}$ corresponds to nonpropagating (evanescent) waves and makes no contribution to the resulting image. This restriction results in loss of information and hence limits the spatial resolution of a lens. If one can make use of this exponentially decaying component in the process of image formation, a loss-less image can be achieved. Pendry reported that a parallel-sided slab of material with the property of negative refractive index and permeability, i.e., $\varepsilon=-1$ and $\mu=-1$, can realize this, together with focusing the far-field [110].

Taking into account of the matching wave field at the interface, the transmission coefficient can be determined. To calculate transmission through both surfaces of the slab, multiple scattering is considered. In the extreme near-field limit, the calculated transmission coefficient tells us that the medium can cancel the decay of evanescent wave components. In practice this limit can never be achieved because of imperfection of real materials which will always absorb some radiation resulting in a small imaginary component of $\varepsilon$, i.e., $\varepsilon=-1+i \delta$, and only the part of wave field which satisfies

$$
\delta<\exp \left(-\sqrt{k_{x}^{2}+k_{y}^{2}} d\right)
$$

will be transmitted through. Here, $d$ represents the slab thickness. This limits the resolution to

$$
\Delta=\frac{2 \pi}{k_{\max }} \approx \frac{2 \pi d}{|\ln \delta|} .
$$

Pendry also proposed a multilayer system of metal and dielectric thin film to realize strong absorption and to enhance the resolution [111].

The real superlens has already been fabricated experimentally by some groups [111-119]. Imaging through a superlens essentially requires the object as well as the image to be within the range of near-field. Zhang and his group [120] recorded the image on photoresist and read it by AFM. The super lens in this case was a $35 \mathrm{~nm}$ thick silver film and the wavelength of excitation light was $365 \mathrm{~nm}$ (not far from the surface plasmon resonance of silver $3.8 \mathrm{eV}$ or $326 \mathrm{~nm}$ ). Superlens with magnifying function has been proposed by Zhang and Smolyaninov independently with concentric ring structures [121,122].

Recently, Kawata et al. have proposed a plasmonic lens made of multilayered arrays of silver nanorods [123]. In their proposal, they showed color imaging of subwavelength objects, where the image can be transferred to long distance without significant loss and can be magnified for its far-field observation.

The image of the letter A in Figure 8a was calculated using a three dimensional FDTD method by taking account of the complex dielectric function of silver. The blurring of pixels in the image was accounted for by the point-spread function in the imaging process. The 50-nmlong nanorods with a diameter of $20 \mathrm{~nm}$ were arranged in hexagonal layers with $10 \mathrm{~nm}$ gap between the layers. The nanorods within arrays with a pitch of $40 \mathrm{~nm}$ guarantees no crosstalk between neighboring rods. In this arrangement, the object source excites the resonance modes of local plasmons in the rods of the first layer, which couple with resonance mode of local plasmons in the rods of the second layer at the nanogap between the two layers and then to the third layer in the same fashion, to finally provide an image at the other end of the third layer. The local plasmons are excited in each layers individually through their coupling at the gaps. Therefore, one would expect the effective resonance response to be closer to that of a 
(a)

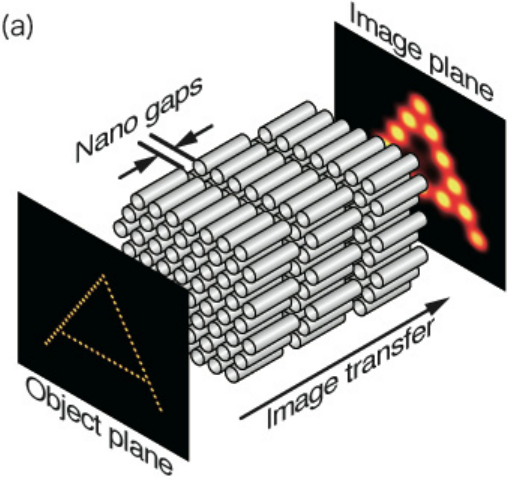

(b)

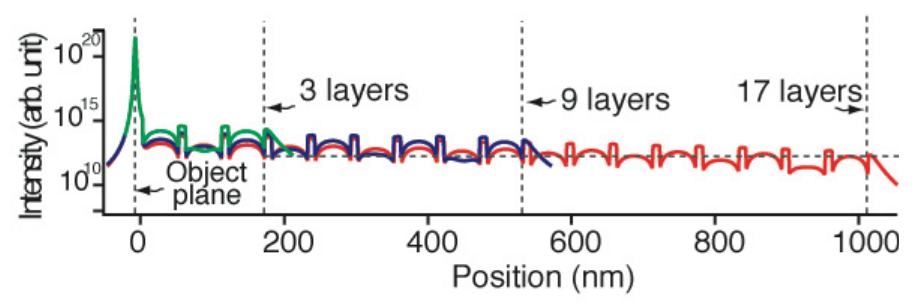

Fig. 8. (Color online) Plasmonic superlens: (a) the basic concept of the stacked arrangement of nanorod arrays for long distance image transfer, (b) the intensity profile across 3 (green line), 9 (blue line) and 17 (red line) layer stacked arrays showing that neither intensity nor the point spread function has any noticeable dependence on distance at least up to micrometer scale distances.

$50 \mathrm{~nm}$ rod, rather than that of the total length of the nanolens. In order to confirm the long distance transfer of an image without suffering significant loss, they also simulated the transfer of light energy for an increasing number of layers. Figure 8b presents a comparison of simulation results for the intensity distributions along the length of the nanolens for cases, in which the nanolens comprise 3,9 and 17 layers of nanorod array. The total length of the device in 17-layer case is about $1 \mu \mathrm{m}$. There is no downward trend of the intensity line profile with increasing length of nanorods, confirming that there is no significant loss of energy along the length of the nanolens. Furthermore, there is no recognizable blurring of the image even after a distance of up to microns. This is because the energy is transferred across the nanolens, through the resonance modes of local plasmons and their interactions at the gap through the evanescent field. There are no long propagations of either plasmons or electromagnetic field in the process of image formation, which minimizes the propagation and radiation related losses. This method also has a magnification function as ordinary lenses have. When the stacked layers are tapered at a certain angle, it is possible to have a large pixel-to-pixel distance at the image plane, while keeping the subwavelength pixel-to-pixel distance at the object plane constant. This efficiently works as the magnification and allows one to record an image of subwavelength resolution using diffraction-limited optics.

\section{Conclusion}

In this article, we have reviewed scanning near-field imaging and spectroscopy techniques in the whole along with three basic properties of surface plasmons; plasmonic nano-structures, polarizations and resonance wavelengths. The near-field probe acts as a nano-light source just like nano-antennas, which enhances the optical emission rate and controls the emission directions. As for designing the plasmonic nanostructures, it has been demonstrated that the lightning rod effect and gap structures are quite efficient to strongly enhance the near-field signals thereby improving greatly the detection sensitivity. Image dipole effect between the probe and the substrate also realize the efficient detection of near-field signals. A polarizationcontrolled measurement, which is unique to ANSOM, is also reviewed. The polarization measurement can be a powerful tool for characterization of chemical composition, molecular orientation and assembled structures. It can also provide a convenient way to separate the nearfield signal from strong far-field background. The information one can obtain from plasmonic nano-imaging strongly depends on the wavelength of light one is using, combined with spectroscopic techniques such as Raman scattering, infrared absorption, fluorescence emission, $\mathrm{THz}$ spectroscopy, etc. The method of tuning plasmon resonance for each wavelength ranges were presented and discussed. Most recent applications of ANSOM in bioscience, single molecule detections are also introduced together with some novel near-field measurement techniques such as pressure dependent measurements, temperature control, and electron emission at the probe apex. A plasmonic superlens that is completely different approach to overcome the limited spatial resolution of a conventional lens was also presented.

The final target of this technique is to image nano-scale object through their spectroscopic information. Many new ideas are developed in ANSOM families, however, the image quality remains somewhat limited due to the stability of the experimental system or due to the lack of signal sensitivity. Since these techniques are done by a "nanoprobe", one has a freedom to choose a particular position on the sample, which means they can potentially be applied for true nano-scale imaging. The short-range issue for the ANSOM is to improve the detection stability and sensitivity, which include the modification of scanning probe microscope and probe fabrications. Though the idea of nano-probe imaging has been shown by Synge almost 100 years ago, it is only recently that the ANSOM was developed experimentally. However, the speed of experimental improvement of ANSOM is enormous, and what we have introduced here is only a part of the recent 
achievements. Imaging through plasmonic nano-probe has a promising future in optics, chemistry, biology, as well as material or semiconductor industries.

\section{References}

1. M. Born, E. Wolf, Principles of Optics, 7th edn. (Cambridge, 1999)

2. S. Kawata, H. Sun, T. Tanaka, K. Takada, Nature 412 $697(2001)$

3. S.W. Hell, J. Wichmann, Opt. Lett. 19, 780 (1994)

4. D.W. Pohl, W. Denk, M. Lanz, Appl. Phys. Lett. 44, 651 (1984)

5. A. Harootunian, E. Betzig, M. Isaacson, A. Lewis, Appl. Phys. Lett. 49, 674 (1986)

6. Near-Field Optics and Surface Plasmon Polaritons, edited by S. Kawata (Springer, Berlin, 2001)

7. E.H. Synge, Phil. Mag. 6, 356 (1928)

8. G. Binnig, H. Rohrer, Helv. Phys. Acta 55, 726 (1982)

9. D.P. Tsai, A. Othonos, M. Moskovits, D. Uttamchandani, Appl. Phys. Lett. 64, 1768 (1994)

10. C.L. Jahncke, M.A. Paesler, H.D. Hallen, Appl. Phys. Lett. 67, 2483 (1995)

11. E. Betzig, R.J. Chichester, Science 262, 1422 (1993)

12. X.S. Xie, R.C. Dunn, Science 265, 361 (1994)

13. W.P. Ambrose, P.M. Goodwin, J.C. Martin, R.A. Keller, Science 265, 364 (1994)

14. F. Zenhausern, M.P. O'Boyle, H.K. Wickramasinghe, Appl. Phys. Lett. 65, 1623 (1994)

15. Y. Inouye, S. Kawata, Opt. Lett. 19, 159 (1994)

16. T. Sugiura, T. Okada, Y. Inouye, S. Kawata, Opt. Lett. 22, 1663 (1997)

17. L. Novotny, E.J. Sánchez, X.S. Xie, Ultramicroscopy 71 , 21 (1998)

18. E. Sánchez, L. Novotny, X.S. Xie, Phys. Rev. Lett. 82 , 4014 (1999)

19. N. Hayazawa, Y. Inouye, Z. Sekkat, S. Kawata, Opt. Commun. 183, 333 (2000)

20. R.M. Stöckle, Y.D. Suh, V. Deckert, R. Zenobi, Chem. Phys. Lett. 318, 131 (2000)

21. K. Kneipp, Y. Wang, H. Kneipp, L.T. Perelman, I. Itzkanm, R. Dasari, Phys. Rev. Lett. 78, 1667 (1997)

22. Applied Scanning Probe Methods, edited by B. Bhushan, H. Fuchs, S. Kawata, Vol. 6 (Springer-Verlag, Heidelberg, 2007)

23. G.P. Wiedrrecht, Eur. J. Appl. Phys. 28, 3 (2004)

24. F. Zenhausern, M.P. O'Boyle, H.K. Wickramasinghe, Appl. Phys. Lett. 65, 1623 (1994)

25. Y. Inouye, N. Hayazawa, K. Hayashi, Z. Sekkat, S. Kawata, Proc. SPIE Int. Soc. Opt. Eng. 3791, 40 (1999)

26. A. Hartschuh, E.J. Sánchez, X.S. Xie, L. Novotny, Phys. Rev. Lett. 90, 095503 (2003)

27. M.S. Anderson, W.T. Pike, Rev. Sci. Instrum. 73, 1198 (2002)

28. W.X. Sun, Z.X. Shen, Ultramicroscopy 94, 237 (2003)

29. D. Mehtani, N. Lee, R.D. Hartschuh, A. Kisliuk, M.D. Foster, A.P. Sokolov, J.F. Maguire, J. Raman Spectrosc. 36, 1068 (2005)

30. Y. Saito, M. Motohashi, N. Hayazawa, M. Iyoki, S. Kawata, Appl. Phys. Lett. 88, 143109 (2006)

31. Y. Saito, M. Motohashi, N. Hayazawa, S. Kawata, J. Microsc. 229, 217 (2008)
32. G.M. Lerman, A. Israel, A. Lewis, Appl. Phys. Lett. 89, $223122(2006)$

33. J.J. Greffet, Science 308, 1561 (2005)

34. P. Royer, D. Barchiesi, G. Lerondel, R. Bachelot, Phil. Trans. R. Soc. Lond. A 362, 821 (2004)

35. T.H. Taminiau, F.D. Stefan, F.B. Segrink, N.F. Van Hulst, Nat. Photon. 2, 234 (2008)

36. M. Futamata, F. Maruyama, M. Ishikawa, J. Phys. Chem. B 108, 13119 (2004)

37. K. Imura, H. Okamoto, M.K. Hossain, M. Kitajima, Nano Lett. 6, 2173 (2005)

38. D.P. Fromm, A. Sundaramurthy, P.J. Schuck, G.S. Kino, W.E. Moerner, Nano Lett. 4, 957 (2004)

39. P.J. Schuck, D.P. Fromm, A. Sundaramurthy, G.S. Kino, W.E. Moerner, Phys. Rev. Lett. 94, 017402 (2006)

40. E.M. Purcell, Phys. Rev. 69, 681 (1946)

41. L. Rogibete, F. Kaminski, M. Agio, V. Sandoghdar, Opt. Lett. 32, 1623 (2007)

42. B.C. Buchler, T. Kalkbrenner, C. Hettich, V. Sandoghdar, Phys. Lev. Lett. 95, 063003 (2005)

43. B. Pettinger, K.F. Domke, D. Zhang, R. Schuster, G. Ertl, Phys. Rev. B 76, 113409 (2007)

44. P. Verma, K. Yamada, H. Watanabe, Y. Inouye, S. Kawata, Phys. Rev. B 73, 045416 (2006)

45. T. Yano, T. Ichimura, A. Taguchi, N. Hayazawa, P. Verma, Y. Inouye, S. Kawata, Appl. Phys. Lett. 91, 121101 (2007)

46. A. Cvitkovic, N. Ocelic, J. Aizpurua, R. Guckengerger, R. Hillenbrand, Phys. Rev. Lett. 97, 060801 (2006)

47. Z.H. Kim, S.R. Leone, J. Phys. Chem. B 110, 19804 (2006)

48. J.D. Jackson, Classical electrodynamics (Wiley, 1963)

49. K.G. Lee, K.J. Ahn, H.W. Kihm, J.S. Ahn, T.K. Kim, S. Hong, Z.H. Kim, D.S. Kim, Opt. Expr. 16, 10641 (2008)

50. Topics in Applied Physics, edited by S. Kawata, Vol. 81 (Springer-Verlag, Berlin, 2001), p. 15

51. N. Hayazawa, Y. Saito, S. Kawata, Appl. Phys. Lett. 85, 6239 (2004)

52. K.G. Lee, H.W. Kihm, J.E. Kihm, W.J. Choi, K. Kim, C. Ropers, D.J. Park, Y.C. Yoon, S.B. Choi, D.H. Woo, J. Kim, B. Lee, Q.H. Park, C. Lienau, D.S. Kim, Nat. Photon. 1, 53 (2007)

53. G. Turrell, J. Raman Spectrosc. 15, 103 (1984)

54. Y. Saito, M. Kobayashi, D. Hiraga, S. Kawano, K. Fujita, N.I. Smith, Y. Inouye, S. Kawata, J. Raman Spectrosc. 39, 1643 (2008)

55. R.J. Moerland, T.H. Taminiau, L. Novotny, N.F. van Hulst, L. Kuipers, Nano Lett. 8, 606 (2008)

56. Y. Saito, N. Hayazawa, H. Kataura, T. Murakami, K. Tsukagoshi, Y. Inouye, S. Kawata, Chem. Phys. Lett. 410, 136 (2005)

57. A.L. Demming, F. Festy, D. Richards, J. Chem. Phys. 122, 184716 (2005)

58. H.I. Ahrach, R. Bachelot, A. Vial, G. Lerondel, J. Plain, P. Royer, Phys. Rev. Lett. 98, 107402 (2007)

59. P. Moon, D.E. Spencer, Field theory for engineers (Van Nost. Reinhold, 1961)

60. D. Molenda, G.C. des Francs, U.C. Fisher, N. Rau, N. Naber, Opt. Expr. 26, 10688 (2005)

61. P.G. Gucciardi, M. Lopes, R. Deturche, C. Julien, D. Barchiesi, M.L. de la Chapelle, Nanotechnology 19, 215702 (2008) 
62. R. Ossikovski, Q. Nguyen, G. Picardi, Phys. Rev. B 75, 045412 (2007)

63. M. Motohashi, N. Hayazawa, T. Alvarado, S. Kawata, J. Appl. Phys. 103, 034309 (2008)

64. C.F. Bohren, D.R. Hoffman, Absorption and scattering of light by small particles (Wiley, New York, 1983)

65. X. Cui, W. Zhang, B.S. Yeo, R. Zenobi, C. Hafner, D. Erni, Opt. Expr. 15, 8309 (2007)

66. B.S. Yeo, W. Zhang, C. Vannier, R. Zenobi, Appl. Phys. 60, $1142(2006)$

67. A. Ono, K. Masui, Y. Saito, T. Sakata, A. Taguchi, M. Motohashi, T. Ichimura, H. Ishitobi, A. Tarun, N. Hayazawa, P. Verma, Y. Inouye, S. Kawata, Chem. Lett. 37, $122(2008)$

68. C.H. Chou, C.D. Chen, C.R.C. Wang, J. Phys. Chem. B 109, 11135 (2005)

69. Y. Yang, S. Matshbara, M. Nogami, J. Shi, W. Huang, Nanotechnology 17, 2821 (2006)

70. J. Ye, C. Chen, W. Van Roy, P. Van Dorpe, G. Maes, G. Borghs, Nanotechnology 19, 1 (2008)

71. A. Bouhelier, R. Bachelot, G. Lerondel, S. Kostcheev, P. Royer, G.P. Wiederrecht, Phys. Rev. Lett. 95, 267405 (2005)

72. E. Prodan, C. Radloff, N.J. Halas, P. Nordlander, Science 302, 419 (2003)

73. E. Prodan, P. Nordlander, J. Chem. Phys. 120, 5444 (2004)

74. A. Oubre, P. Nordlander, J. Phys. Chem. B 109, 10042 (2005)

75. S. Lal, N.K. Grady, J. Jundu, C.S. Levin, J.B. Lassoter, N.J. Halas, Chem. Soc. Rev. 37, 898 (2008)

76. J. Kundu, F. Le, P. Nordlander, N.J. Halas, Chem. Phys. Lett. 452, 115 (2008)

77. A. Huber, N. Ocelic, D. Kazantsev, R. Hillenbrand, Appl. Phys. Lett. 87, 081103 (2005)

78. M. Brehm, T. Taubner, R. Hillenbrand, F. Keilmann, Nano Lett. 6, 1307 (2006)

79. H.T. Chen, S. Kraatz, G.C. Cho, R. Kersting, Phys. Rev. Lett. 93, 267401 (2004)

80. M. Walther, G.S. Chambers, Z. Liu, M.R. Freeman, F. Hegmann, Opt. Soc. Am. B 22, 2357 (2005)

81. R. Lecaque, S. Gresillon, N. Barbey, R. Peretti, J.C. Rivoal, C. Boccara, Opt. Commun. 262, 125 (2005)

82. R. Lecaque, S. Gresillon, C. Boccara, Opt. Expr. 16, 4731 (2008)

83. A.J.L. Adam, N.C.J. van der Valk, P.C.M. Planken, J. Opt. Soc. Am. 24, 1080 (2007)

84. H.T. Chen, R. Kersting, G.C. Cho, Appl. Phys. Lett. 83, 3009 (2003)

85. Z.Q. Tian, Z.L. Yang, B. Ren, D.Y. Wu, Top. Appl. Phys. 103, $125(2006)$

86. C.A. Brewer, F. Brizuela, P. Wachulak, D.H. Martz, W. Chao, E.H. Anderson, D.T. Attwood, V. Vinogradov, I.A. Artyukov, A.G. Ponomareko, V.V. Kondratenko, M.C. Marconi, J.J. Rocca, C.S. Menoni, Opt. Lett. 33, 518 (2008)

87. G. Vaschenko, F. Brizuela, M. Grisham, H. Marconi, M.C. Marconi, J.J. Rocca, Opt. Expr. 30, 2095 (2005)

88. A. Hartschuh, H. Qian, A.J. Meixner, L. Novotny, Surf. Int. Anal. 38, 1472 (2006)

89. M. Koopman, A. Cambi, B.I. de Bakker, B. Joosten, C.G. Figdor, N.F. van Hulst, M.F. Garcia-Parajo, FEBS Lett. $\mathbf{5 7 3 , 6}(2004)$
90. C. Hoppener, J.P. Siebrasse, R. Peters, U. Kubitscheck, A. Naber, Biophys. J. 88, 3681 (2005)

91. C. Hoppener, L. Novotny, Nano Lett. 8, 642 (2008)

92. B.S. Yeo, S. Madler, T. Schmid, W. Zhang, R. Zenobi, J. Chem. Phys. C 112, 4867 (2008)

93. A. Rasmussen, V. Deckert, J. Raman Spectrosc. 37, 311 (2006)

94. T. Ichimura, H. Watanabe, Y. Morita, P. Verma, S. Kawata, Y. Inouye, J. Phys. Chem. 111, 9460 (2007)

95. Y.J. Yu, H. Noh, G.S. Jeon, H.R. Noh, Y. Arakawa, W. Jhe, Appl. Phys. Lett. 91, 041117 (2007)

96. R. Matsui, P. Verma, T. Ichimura, Y. Inouye, S. Kawata, Appl. Phys. Lett. 90, 061906 (2007)

97. M.B. Raschke, L. Molina, T. Elsaesser, D.H. Kim, W. Knoll, K. Hinrichs, Chem. Phys. Chem. 6, 2197 (2005)

98. Y. Maruyama, M. Ishikawa, M. Futamata, J. Phys. Chem. B 108, 673 (2004)

99. P. Anger, P. Bharadwaj, L. Novotny, Phys. Rev. Lett. 96, $113002(2006)$

100. K. Vasilev, W. Knoll, M. Kreiter, J. Chem. Phys. 120, 3439 (2004)

101. I.A. Larkin, M.I. Stockman, M. Achermann, V.I. Klimov, Phys. Rev. B 69, 121403 (2004)

102. A.O. Govorov, J. Lee, N.A. Kotov, Phys. Rev. B 76, 125308 (2007)

103. S. Kuhn, U. Hakanson, L. Rogobete, V. Sandoghdar, Phys. Rev. Lett. 97, 017402 (2006)

104. W. Zhang, B.S. Yeo, T. Schmid, R. Zenobi, J. Chem. Phys. C 111, 1733 (2007)

105. T. Yano, Y. Inouye, S. Kawata, Nano. Lett. 6, 1269 (2006)

106. C. Ropers, D.R. Solli, C.P. Schulz, C. Lienau, T. Elsaesser, Phys. Rev. Lett. 98, 043907 (2007)

107. I. Gerhardt, G. Wrigge, M. Agio, P. Bushev, G. Zumofen, V. Sandoghdar, Opt. Lett. 32, 1420 (2007)

108. Y.D. Wilde, F. Formanek, R. Carminati, B. Gralak, P.A. Lemoine, K. Joulain, J.P. Mulet, Y. Chen, J.J. Greffet, Nature 444, 740 (2006)

109. P.O. Chapuis, J.J. Greffet, K. Joulian, S. Volz, Nanotechnology 17, 2978 (2006)

110. J.B. Pendry, Phys. Rev. Lett. 85, 3966 (2000)

111. J.B. Pendry, S.A. Ramakrishna, Physica B 338, 329 (2003)

112. G. Shvets, S. Trendafilov, J.B. Pendry, A. Sarychev, Phys. Rev. Lett. 99, 053903 (2007)

113. X.S. Rao, C.K. Ong, Phys. Rev. E 68, 067601 (2003)

114. V.A. Podplskiy, E.E. Narimanov, Opt. Lett. 30, 75 (2005)

115. I.A. Larkin, M.I. Stockman, Nano Lett. 5, 339 (2005)

116. T. Taubner, D. Korobkin, Y. Urzhumov, G. Shvets, R. Hillenbrand, Science 313, 1595 (2006)

117. R.J. Moerland, N.F. van Hulst, Opt. Expr. 13, 1604 (2005)

118. A. Ono, J. Kato, S. Kawata, Chem. Phys. Lett. 95, $267407(2005)$

119. R. Ruppin, O.J.F. Martin, J. Chem. Phys. 121, 11358 (2004)

120. N. Fang, H. Lee, C. Sun, X. Zhang, Science 308, 534 (2005)

121. X. Zhang, Z. Liu, Nat. Mater. 7, 435 (2008)

122. I.I. Smolyaninov, Y.J. Hung, C.C. Davis, Science 315, 1699 (2007)

123. S. Kawata, A. Ono, P. Verma, Nat. Photon. 2, 438 (2008) 